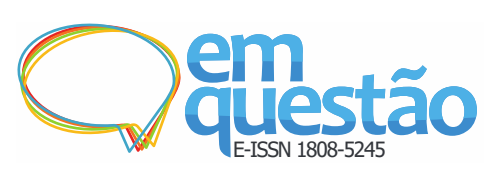

\title{
Análise da longevidade e do tamanho das coautorias acadêmicas: os caminhares na ciência brasileira
}

\author{
Andréia Cristina dos Santos Gusmão \\ Mestre; Universidade Federal do ABC, Santo André, SP, Brasil; \\ andreia.gusmao@ufabc.edu.br; ORCID: https://orcid.org/0000-0003-0523-6146 \\ Solange Maria dos Santos \\ Doutora; Scientific Electronic Library Online, São Paulo, SP, Brasil; \\ solange.santos@scielo.org; ORCID: https://orcid.org/0000-0002-5067-6362 \\ Jesús Pascual Mena-Chalco \\ Doutor; Universidade Federal do ABC, Santo André, SP, Brasil; \\ jesus.mena@ufabc.edu.br; ORCID: https://orcid.org/0000-0001-7509-5532
}

Resumo: O presente artigo propõe um método de detecção, identificação e visualização da longevidade (tempo) e do tamanho da coautoria (número de pessoas) na ciência brasileira, possibilitando a análise dos caminhares dos pesquisadores de acordo com as áreas do conhecimento. Os dados utilizados referem-se a todos os doutores ativos em 2019 registrados na Plataforma Lattes. O método empregado permite observar todos os possíveis agrupamentos de pesquisadores na colaboração em coautoria ao longo do tempo. Os resultados apresentados evidenciam que grupos menores têm uma longevidade maior em relação aos grupos de tamanhos maiores. Este artigo caracteriza de maneira inédita os caminhares (em função da longevidade e tamanho) dos pesquisadores brasileiros, permitindo observar o atuar de cada pesquisador não de forma isolada/independente mas de forma grupal/dependente da interação colaborativa e social, como a ciência é realizada na atualidade.

Palavras-chave: Colaboração científica; Coautoria; Longevidade; Caminhar acadêmico.

\section{Introdução}

A colaboração científica é vista como virtude quando entendida como dois ou mais cientistas trabalhando juntos em um projeto de pesquisa, compartilhando recursos intelectuais, econômicos e/ou físicos para atingir o objetivo comum de produzir novos conhecimentos científicos (KATZ; MARTIN, 1997; VANZ; 
STUMPF, 2010). Nesse sentido, políticas públicas incentivam ativamente a colaboração tanto em nível individual como em nível institucional. Em vista disso, a colaboração tem se tornado uma constante e não mais uma exceção (KATZ; MARTIN, 1997), de tal forma que o aumento da colaboração nos últimos anos é um dos fenômenos mais visíveis nos estudos da produção científica.

Ainda no que se refere à colaboração científica é importante observar que, muitas vezes, esta aparece na literatura relacionada à coautoria e por essa razão, estas interações são frequentemente consideradas sinônimos, embora a coautoria seja apenas uma faceta da colaboração científica, pois ela não mede a colaboração na sua totalidade e complexidade. Nesse sentido, Katz e Martin (1997) destacam que a coautoria não pode ser assumida como sinônimo de colaboração uma vez que nem toda a colaboração resulta em artigo publicado e nem sempre a coautoria indica colaboração.

$\mathrm{Na}$ busca por uma definição mais operacional de colaboração, Katz e Martin (1997) ponderam que poderia ser considerado como colaborador qualquer indivíduo que oferecesse uma contribuição válida para uma determinada pesquisa. Desse modo, propuseram alguns critérios para distinguir os colaboradores dos pesquisadores sem contribuição. Podem ser considerados colaboradores:

a) aqueles que trabalham juntos durante toda a duração de um projeto de pesquisa, aqueles que trabalharam por um longo período dentro da duração do projeto ou que fizeram contribuições frequentes ou substanciais;

b) aqueles que colaboraram na proposta original de um projeto de pesquisa; e

c) os responsáveis por um ou alguns dos principais elementos da pesquisa, por exemplo, o projeto experimental, construção de equipamentos de pesquisa, execução do experimento, análise e interpretação dos dados (KATZ; MARTIN, 1997, p.12). 
Além dos aspectos que caracterizam um colaborador, é importante considerar também que a ciência vem crescendo globalmente. Desde o início do século XXI, o gasto mundial em pesquisa e desenvolvimento praticamente dobrou, e com isso, as publicações vêm crescendo ano após ano (THE ROYAL SOCIETY, 2011). Nesse contexto, o caráter internacional do avanço do conhecimento tem intensificado a colaboração científica fazendo com que esta seja uma das principais características da atividade científica, trazendo à tona a importância da interdisciplinaridade e do intercâmbio de conhecimento entre os membros da comunidade científica.

Segundo Leta, Thijs e Glänzel (2013) atualmente é impensável que possa ocorrer algum avanço da ciência sem qualquer nível de cooperação pois a Ciência atingiu um nível de maturidade que requer esforço coletivo que congrega diferentes atores, competências e habilidades para seu desenvolvimento (ADAMS, 2012; LEYDESDORFF et al., 2013).

Dada a complexidade atual do empreendimento científico, dificilmente um único pesquisador possui o conhecimento ou habilidades para lidar com questões científicas complexas tornando a necessidade de colaboradores interdisciplinares muito mais comum e requisitada (HAINES; GODLEY; HAWE, 2011), além de tornar necessário o desenvolvimento de diversos métodos buscando a colaboração entre pesquisadores (SIDONE; HADDAD; MENA-CHALCO, 2016). Diante desse cenário, a colaboração científica é cada vez mais importante e tem sido extensivamente investigada por pesquisadores em várias disciplinas, como no presente estudo que reúne pesquisadores da Ciência da Informação e Ciência da Computação.

Neste trabalho, analisamos a longevidade de uma coautoria acadêmica, aqui entendida como o tempo de duração da colaboração, em anos, da coautoria de um grupo de pesquisadores. De forma análoga, definimos como tamanho de uma coautoria acadêmica o número de pesquisadores que participam de forma concomitante na elaboração de uma publicação. Com base nestas definições, podemos observar o que denominamos "caminhar de um coautor" que é determinado pela longevidade e tamanho de sua coautoria. 
Diante do exposto, o objetivo deste estudo é caracterizar, de forma inédita, os caminhares dos pesquisadores brasileiros por área do conhecimento e por idade acadêmica, através de uma análise quantitativa considerando o tamanho e a longevidade das coautorias acadêmicas.

\section{Procedimentos metodológicos}

Antes de seguirmos à apreciação dos dados, apresentamos uma síntese da metodologia, pois este artigo faz parte de um empreendimento maior, envolvendo várias etapas e procedimentos metodológicos. No entanto, em razão da limitação de espaço aqui necessária, a apresentação da metodologia detalhada em sua totalidade nos deixaria menor espaço para aquilo que compreendemos ser a contribuição principal deste artigo que é a análise da longevidade e tamanho de coautorias que caracterizam algumas áreas da ciência brasileira.

\subsection{Tempo de colaboração: longevidade da coautoria}

Sabe-se que os autores podem realizar muitas colaborações ao longo de suas carreiras acadêmicas. No entanto, a duração da colaboração entre pesquisadores pode variar de acordo com as temáticas, "maturidade acadêmica" e tipo de vínculos entre os pesquisadores que colaboram, dentre outros fatores.

Devido ao problema de sobrecarga de informações, não é fácil para os acadêmicos encontrarem novos colaboradores, especialmente um colaborador vitalício. WANG et al. (2019) investigaram se era possível prever/identificar colaboradores vitalícios com base nos estágios iniciais de colaborações, dado um acadêmico e seus colaboradores, com o objetivo de prever se existia uma colaboração que atingiria o valor limite de colaborador vitalício em um tempo futuro. Para tanto, considera-se 30 anos de colaboração para determinar se um colaborador é vitalício.

$\mathrm{Na}$ literatura alguns estudos prévios contribuem para a análise do tempo de colaboração e do tamanho do grupo de pesquisadores que colaboram (TUESTA et al., 2015; TSAI; LIN, 2016; WANG et al., 2019; GAO et al., 2020), no entanto, nesses trabalhos correlatos, os aspectos de tempo e tamanho 
são independentes e por isso, propomos analisar o "caminhar de um coautor", considerando tanto a sua longevidade, quanto o tamanho da coautoria.

\subsection{Tamanho do grupo}

No trabalho de Tuesta et al. (2015), os autores examinaram a relação entre os pesquisadores da área de Ciências Exatas e da Terra (em oito subáreas) inscritos na Plataforma Lattes de 1981 a 2010, com o diferencial de medir a evolução temporal do índice de dependência entre orientador e orientado. Os autores encontraram evidências de que existe uma correlação positiva entre o tempo da relação orientador e orientado com a produtividade do orientado. No entanto, a evolução da relação entre os pares diminui ao longo do período em estudo. Isso sugere que o doutorando obtém mais independência em relação ao seu orientador ao longo dos anos e/ou incrementa seu grupo de colaboração de pesquisa.

Em um estudo semelhante realizado recentemente, Gao et al. (2020) adotaram uma nova abordagem baseada na rede de colaboração. A rede de colaboração científica está em constante alteração ao longo do tempo, novas relações de colaboração podem aparecer e dois pesquisadores podem parar de colaborar entre si, portanto, é necessário considerar a rede dinâmica para identificar o relacionamento. Se construirmos a rede de colaboração em períodos diferentes, a rede é totalmente diferente.

Diante disso, Gao et al. (2020) propuseram um método que visa identificar os relacionamentos orientador e orientado em redes de colaboração científica construídas a partir de uma biblioteca digital ou conjuntos de dados acadêmicos.

\subsection{Fonte de informação e coleta de dados}

Para a realização do estudo a Plataforma Lattes (http://lattes.cnpq.br/) foi a principal fonte de informação empregada. A escolha desta fonte se deu por ser um repositório que fornece dados de milhões de pesquisadores de diversas áreas de pesquisa e que registra de forma bem completa os dados dos autores, suas publicações e coautorias. A Plataforma Lattes possibilita reunir em um único 
banco de dados informação sobre a trajetória de praticamente toda a comunidade acadêmica brasileira, além de fornecer informações como: código do currículo, nome do autor, nomes dos autores nas citações, área e grande área do conhecimento, lista de publicações, data de defesa do primeiro título de doutor, dentre outros dados.

Por estas características a Plataforma Lattes tem sido frequentemente utilizada em estudos com distintas abordagens de análise e mapeamento de produção e colaboração científica (LANE, 2010; TUESTA et al., 2019; DIGIAMPIETRI et al., 2021).

Em março de 2020 a Plataforma Lattes contava com 6.665.633 pesquisadores cadastrados. Devido ao volume de dados, foi necessário selecionar desse montante grupos representativos para realizar os experimentos. Assim, foram selecionados somente os pesquisadores doutores ativos segundo as áreas do conhecimento. Foram considerados como ativos os pesquisadores que atualizaram seus currículos no período entre 2019 e março de 2020 e foram selecionadas somente as áreas do conhecimento com pelo menos 100 pesquisadores associados. No total, foram considerados 218.048 pesquisadores, de 81 áreas do conhecimento, que contavam com pelo menos cem pesquisadores cadastrados, conforme apresenta a Tabela 2. É importante ressaltar que a Plataforma Lattes oferece o acesso e extração dos currículos em formato XML, assim como as definições do formato (PLATAFORMA LATTES, 2020).

Para determinar o caminhar de um pesquisador de forma individual foram consideradas as publicações do pesquisador realizadas em coautoria registradas na Plataforma Lattes. Foram consideradas as publicações de quatro tipos: artigos em periódicos científicos, trabalhos em congresso, livros e capítulos de livros, sem restrição quanto ao período de publicação, ou seja, foram consideradas também publicações realizadas anteriormente à obtenção do título de doutor do pesquisador que determina sua "idade acadêmica".

O método proposto para gerar os agrupamentos de coautores somente cria grupos de coautores que tenham no mínimo uma publicação. Esse critério foi aplicado por idade acadêmica e por área do conhecimento, utilizando os 
dados bibliográficos dos pesquisadores doutores ativos, registrados na Plataforma Lattes.

\subsection{Etapas}

O percurso metodológico foi realizado de acordo com as 4 etapas descritas a seguir.

\subsubsection{Etapa 1: Identificação dos coautores das publicações}

Foram consideradas publicações em coautoria aquelas realizadas pelo pesquisador em análise, que contavam com pelo menos um coautor. Os coautores foram identificados de forma única, por exemplo, todos os colaboradores que participaram de pelo menos uma publicação. A identificação única independe do número de vezes em que colaboraram e dos veículos nos quais publicaram. Nesta etapa, a principal dificuldade foi identificar os pesquisadores de forma unívoca considerando que o nome dos pesquisadores, principalmente quando abreviados, impõe um desafio adicional ao procedimento de desambiguação.

Cada coautor foi considerado único apenas pela sua grafia (abreviada ou completa). O único tratamento realizado foi remoção de acentos e caracteres especiais. Assim, caso tenha havido um erro na grafia, como por exemplo o nome de um coautor, com ou sem acento, em uma publicação ambos foram igualmente considerados.

\subsubsection{Etapa 2: Identificando os maiores grupos de coautores}

Para cada coautor identificado na Etapa 1, identificamos os coautores que colaboraram juntos nas mesmas publicações também de forma única. $O$ pesquisador analisado não aparece nessa verificação, pois ele colaborou em todas as publicações. Identificamos em cada publicação o número de coautores para determinar qual o maior agrupamento possível entre todas as publicações. 


\subsubsection{Etapa 3: Cálculo de todos os agrupamentos possíveis}

Para cada coautor identificamos qual a publicação com maior número de coautores que determinado coautor colaborou, dessa forma, se um coautor participou de publicações com apenas dois coautores, não há necessidade de verificar um grupo de três pesquisadores com esse coautor. Assim, identificamos todos os grupos possíveis considerando cada coautor da Etapa 1 mais o pesquisador analisado e os seus coautores correspondentes da Etapa 2. Dessa forma fica garantido que todos os agrupamentos tenham pelo menos uma publicação.

A verificação dos possíveis agrupamentos é da ordem da sequência de Mersennse (https://oeis.org/A000225). Por exemplo, para um pesquisador com 4 coautores seriam necessárias $15\left(2^{4}-1\right)$ combinações (como se pode observar na seção 2.5 no número de linhas horizontais na Figura 1). Note que com essa etapa, garantimos que somente fossem geradas as combinações de coautores que tivessem no mínimo uma publicação.

\subsubsection{Etapa 4: Contagem dos agrupamentos}

Para cada tamanho de grupo, contamos em cada agrupamento formado na etapa anterior quantas vezes essa combinação ocorria dentre todas as publicações. Se fosse uma única vez, o caminhar para esse tamanho de grupo era contabilizado em um ano.

Para outros casos, buscamos o ano das publicações que continham a mesma combinação para determinar a longevidade do caminhar, calculada pelo ano mais atual de publicação menos o ano da publicação mais distante. Após essa análise, identificamos o mais longo caminhar por grupo e o mais longo caminhar geral por pesquisador.

\subsection{Exemplo de identificação da longevidade e tamanho das coautorias}

Para exemplificar o método consideramos o currículo do pesquisador $\mathrm{C} 1$ com sete publicações ao longo da sua vida acadêmica, representados por P1, P2, P3, P4, P5, P6 e P7 (Figura 1 superior). Para cada coautor único identificamos os coautores que colaboraram nas mesmas publicações. Por exemplo, na Figura 1 
inferior o coautor $\mathrm{C} 2$ colaborou nas publicações de $\mathrm{C} 1$ com três pesquisadores diferentes: C3 (P2 e P6), C4 (P5) e C5 (P5). Lembrando que o autor C1 não é mostrado na Figura 1 inferior, pois o autor principal colaborou em todas as publicações. O tamanho máximo de grupo para o coautor $\mathrm{C} 2$ é a quantidade de coautores da publicação que conta com maior número de colaboradores em que C2 é coautor. Portanto, o tamanho é 4, pois a publicação P5 tem 4 coautores, enquanto P2 e P6 contam com 3 coautores cada.

Figura 1 - Exemplo de identificação dos caminhares de C1 ao longo dos anos 2016 a 2020. Pi representa a publicação $i(i=1, \ldots, 7)$. C j representa o coautor $j(j=1, \ldots, 5)$.

\begin{tabular}{|c|c|c|c|}
\hline \multirow{8}{*}{ Publicaçoes do coautor C1 } & Publicação & Ano & Coautores \\
\hline & P1 & 2016 & C1, C3, C5 \\
\hline & P2 & 2017 & $\mathrm{C} 1, \mathrm{C} 2, \mathrm{C} 3$ \\
\hline & P3 & 2018 & $\mathrm{C} 1, \mathrm{C} 3$ \\
\hline & P4 & 2018 & $\mathrm{C} 1, \mathrm{C4}$ \\
\hline & P5 & 2020 & $\mathrm{C1}, \mathrm{C} 2, \mathrm{C4}, \mathrm{C5}$ \\
\hline & P6 & 2020 & C1, C2, C3 \\
\hline & P7 & 2020 & $\mathrm{C} 1, \mathrm{C5}$ \\
\hline \multirow{5}{*}{$\begin{array}{l}\text { Maiores grupos de cada } \\
\text { coautor de } \mathrm{C} 1\end{array}$} & Coautor & $\begin{array}{c}\text { Grupo } \\
\text { máximo }\end{array}$ & Coautores \\
\hline & $\mathrm{C} 2$ & 4 (P5) & $\mathrm{C} 3, \mathrm{C} 4, \mathrm{C} 5$ \\
\hline & $\mathrm{C3}$ & $3(P 1, P 2$ e $P 6)$ & $\mathrm{C} 2, \mathrm{C} 5$ \\
\hline & $\mathrm{C4}$ & 4 (P5) & $\mathrm{C} 2, \mathrm{C5}$ \\
\hline & $\mathrm{C5}$ & 4 (P5) & $\mathrm{C} 2, \mathrm{C} 3, \mathrm{C} 4$ \\
\hline
\end{tabular}

Fonte: Elaborada pelos autores.

$\mathrm{Na}$ Figura 2 mostramos os 15 possíveis agrupamentos para cinco coautores com tamanhos de grupos de dois a cinco coautores. Note que nem todos os agrupamentos têm uma publicação correspondente, ou seja, não apresentam um caminhar. Aqui o desafio do método foi reduzir a quantidade de agrupamentos formados de forma que somente fossem gerados os grupos de coautores que tivessem pelo menos uma publicação. Desse modo, a partir do método desenvolvido seriam criados apenas 10 agrupamentos. Como mostra a Figura 2, o caminhar é representado por uma linha reta horizontal e os momentos de colaboração são representados por círculos.

Para o tamanho 3, o caminhar de maior longevidade é de dois anos, para o grupo de coautores representados por $\mathrm{C} 1, \mathrm{C} 2$ e $\mathrm{C} 3$ que colaboraram juntos na publicação P2. 
Observe que, nesse exemplo, para cinco coautores não temos nenhuma publicação, logo, não temos um caminhar de tamanho 5. De acordo com a etapa dois, identificamos que o tamanho máximo de coautores que publicaram em uma mesma publicação é quatro (Figura 1 inferior), portanto, com o método desenvolvido não é necessário gerar os agrupamentos de tamanho igual ao número de coautores, e sim, de tamanho igual ao número de coautores da publicação que contou com mais colaboradores. Assim, não geramos agrupamentos desnecessários que não tinham publicação com esses coautores.

Figura 2 - Longevidade dos caminhares de C1 identificados a partir das publicações.

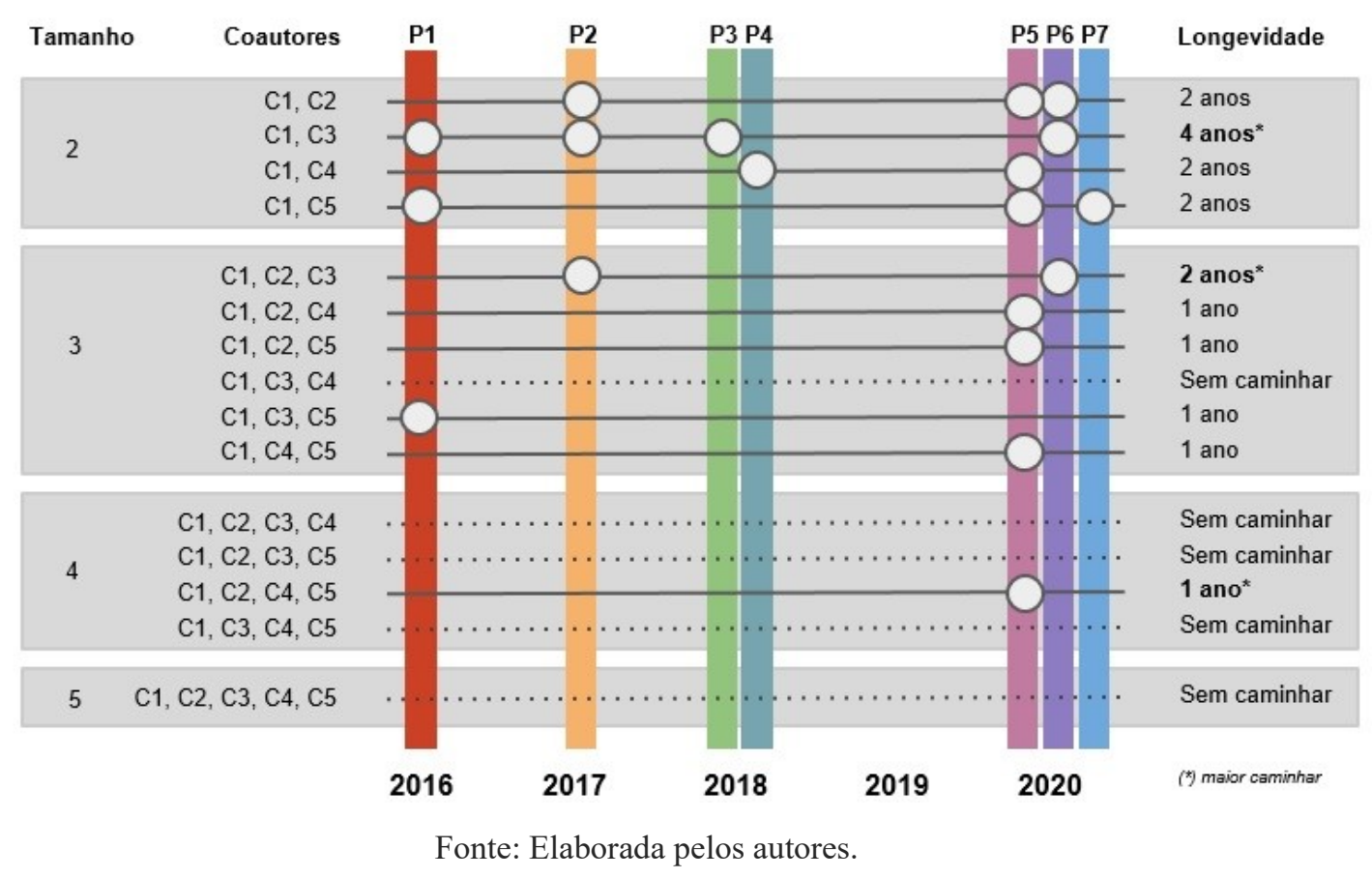

\section{Resultados}

Os resultados foram organizados em três partes, sendo, análise geral dos pesquisadores por área, análise dos caminhares por área do conhecimento, e por idade acadêmica.

\subsection{Análise geral por área do conhecimento}

A análise dos dados apresentados na Tabela 1 mostra que os pesquisadores das áreas de Educação, Direito e Letras foram os que mais publicaram de forma individual (autoria única) ao passo que, os pesquisadores de Física se destacam 
pelo maior número de colaboradores por publicação, sendo essa a área com maior número de publicações com mais de 40 coautores desconsideradas da análise.

A coluna "Maior grupo de coautores" da Tabela 1, mostra que mais de $50 \%$ das áreas analisadas possuem publicações com 40 coautores, apenas as áreas de Engenharia de Minas, Engenharia Naval e Oceânica e Museologia contavam com publicações com o maior grupo de colaboradores inferior a 20 autores. 
Análise da longevidade e do tamanho das coautorias acadêmicas: os caminhares na ciência brasileira

Andréia Cristina dos Santos Gusmão, Solange Maria dos Santos, Jesús Pascual Mena-Chalco

Tabela 1 - Número de pesquisadores e de publicações por área de conhecimento extraídas da Plataforma Lattes até 2020 para determinar tamanho dos grupos

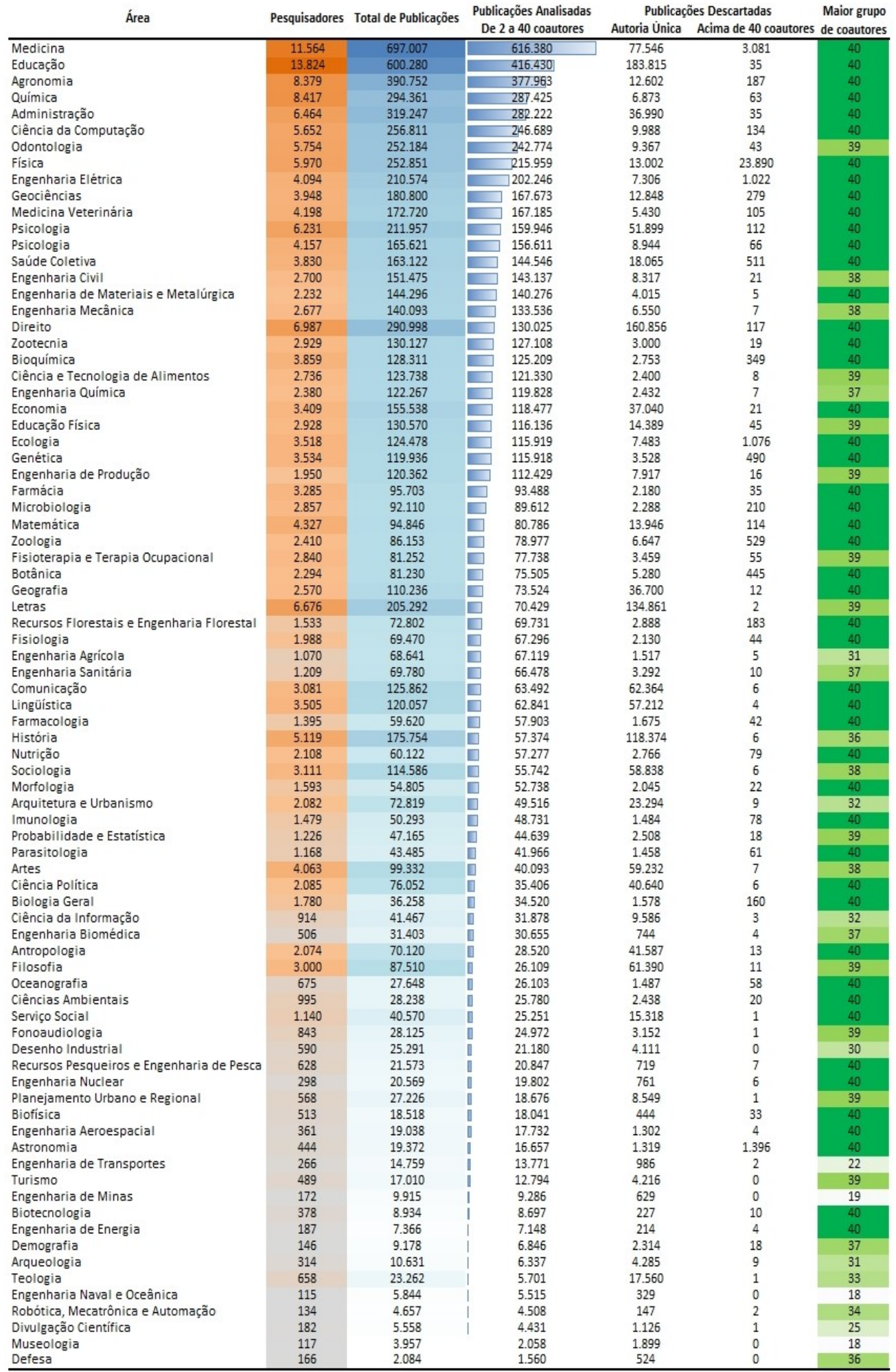

Observação: Tabela ordenada pelo númeo total de publicações de cada área (sem recorte de período de publicação)

Fonte: Elaborada pelos autores. 
As áreas de Medicina (697.007 publicações), Educação (600.280), Agronomia (390.752), Química (294.361), Administração (319.247), Ciência da Computação (256.184), Odontologia (252.184), Física (252.851) e Engenharia Elétrica (210.574) se destacam com mais de 200 mil publicações cada e possuem o maior número de publicações analisadas (Tabela 1). Por outro lado, a área de Direito com 290.998 publicações teve menos de 50\% das publicações analisadas (130.025), já que a maior parte das publicações foram realizadas por apenas um autor. Situação semelhante ocorreu também com os pesquisadores da área de Letras.

No que se refere à quantidade de pesquisadores por área (coluna "Pesquisadores" da Tabela 1), pode-se perceber que algumas áreas se destacam em termos do tamanho de suas comunidades, como é o caso da área de Educação com 13.824 pesquisadores lidera a lista, seguida pela área de Medicina com (11.564 pesquisadores) e pela área de Química (8.417 pesquisadores).

$\mathrm{Na}$ direção oposta temos oito áreas com menos de 150 pesquisadores doutores ativos, é o caso de Engenharia Naval e Oceânica (115 pesquisadores), Museologia (117 pesquisadores) e Demografia (146 pesquisadores) (Tabela 1).

Outros aspectos analisados neste estudo foram a idade acadêmica e as áreas dos pesquisadores, para mostrar o comportamento dos pesquisadores em relação aos grupos de coautores e o tempo em que colaboram juntos.

\subsection{Análise dos caminhares por idade acadêmica}

Consideramos a idade acadêmica de cada pesquisador o intervalo, em número de anos transcorridos, desde a defesa do primeiro título de doutorado. Dividimos a idade acadêmica em cinco categorias: de 0 a 10 anos, 11 a 20 anos, 21 a 30 anos, 31 a 40 anos e acima de 41 anos.

O caminhar de um pesquisador é medido pela quantidade de anos que ele colabora e publica com um determinado coautor. Na Figura 3, estabelecemos essa relação entre o tamanho do grupo de coautores e por quanto tempo esse grupo publicou, para todos os grupos de dois, três, quatro e cinco pesquisadores. 
Para o grupo de dois pesquisadores (Figura 3a), podemos notar que a mediana do caminhar é de três anos para os pesquisadores com idade acadêmica de 0 a 10 anos. Esse número decresce à medida que aumenta a idade acadêmica do pesquisador: de 11 a 20 anos, a mediana é de cinco anos, de 21 a 30 anos, aumenta para oito anos. Para os pesquisadores de maior idade acadêmica, que se enquadram nas duas últimas categorias (31 a 40 anos e acima de 41 anos), a mediana do caminhar segue estável em dez anos, sendo o maior valor entre todas as categorias.

Os dados dos experimentos mostram que grupos menores tendem a publicar juntos por mais tempo. O maior caminhar por idade acadêmica, assim como por área, é para grupos de dois pesquisadores que juntos chegaram a publicar por 46 anos. Essa informação não está sendo mostrada na Figura 3, pois representamos somente os resultados que não são considerados outliers ${ }^{2}$.

Outro aspecto que pôde ser observado sobre a idade acadêmica é que existem caminhares de mais de 20 anos para um grupo de dois pesquisadores com idade acadêmica entre 0 e 10 anos. Cabe destacar que a idade acadêmica, conforme mencionado, contabiliza a quantidade de anos transcorridos a partir do primeiro título de doutor, já as publicações analisadas englobam toda vida acadêmica do pesquisador conforme registrado na Plataforma Lattes, sendo assim, em alguns casos, o caminhar de um pesquisador pode ter início antes mesmo de sua titulação como doutor.

Para o grupo de três pesquisadores (Figura 3b), podemos notar o aumento da mediana do caminhar da mesma forma ocorrida com o grupo de dois pesquisadores (Figura 3a), porém, com a menor longevidade em anos. Para os pesquisadores com idade acadêmica de 0 a 10 anos, a mediana do caminhar é de dois anos, para os pesquisadores de 11 a 20 anos de idade acadêmica são três anos, e quatro anos para os que estão entre 21 a 30 anos. Para os pesquisadores mais experientes, a partir de 31 anos de idade acadêmica, a mediana do caminhar é de cinco anos. Podemos verificar também, para os pesquisadores acima de 41 anos, caminhares bem longos, acima de 30 anos. Para os grupos a partir de quatro pesquisadores (Figura $3 \mathrm{c}$ e $3 \mathrm{~d}$ ), percebemos que a mediana dos “caminhares" são menores. 
Figura 3 - Idade acadêmica e longevidade em anos para grupos de dois, três, quatro e cinco pesquisadores

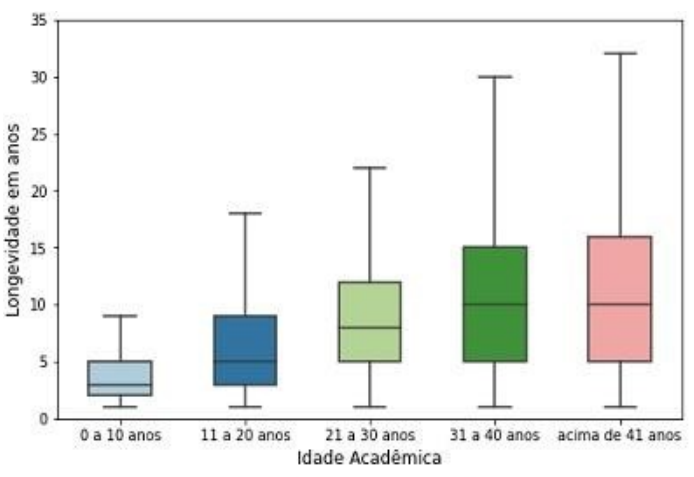

(a) Grupo de dois pesquisadores

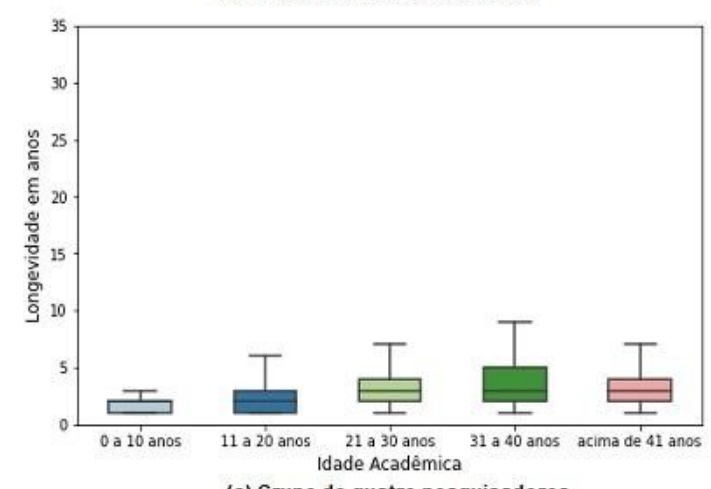

(c) Grupo de quatro pesquisadores

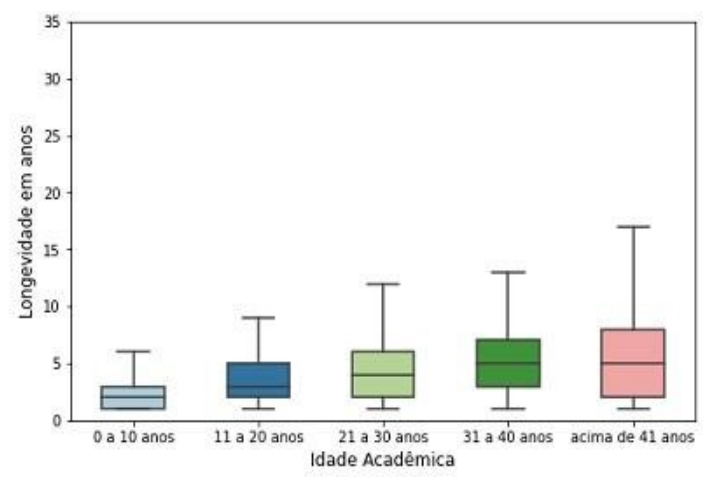

(b) Grupo de três pesquisadores

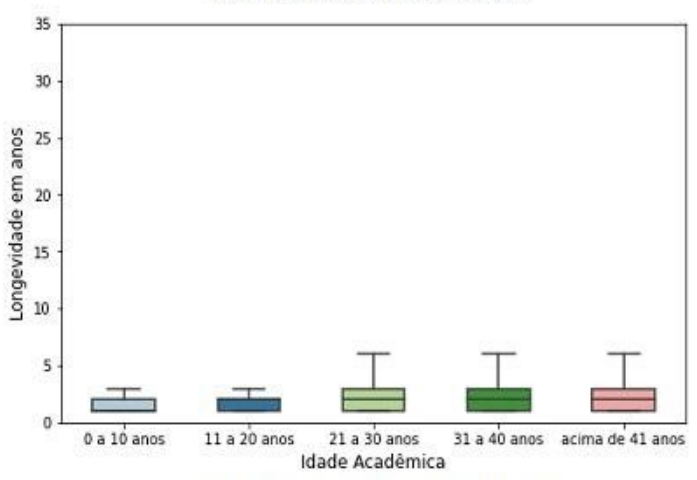

(d) Grupo de cinco pesquisadores

Fonte: Elaborada pelos autores.

\subsection{Análise dos caminhares por área do conhecimento}

Além dos caminhares por idade acadêmica dos pesquisadores, também foram analisados os caminhares por áreas do conhecimento. Nesse sentido, a análise dos caminhares por área do conhecimento (81 áreas para grupos de dois a quarenta pesquisadores) revela que para grupos a partir de seis pesquisadores a mediana do caminhar segue um padrão: quanto maior o grupo, menor é a longevidade. Portanto, grupos menores, apresentam maior longevidade, i.e., tendem a caminhar juntos por mais tempo.

Na Figura 4 mostramos a relação entre a área e a longevidade dos caminhares para todos os grupos de dois pesquisadores. 

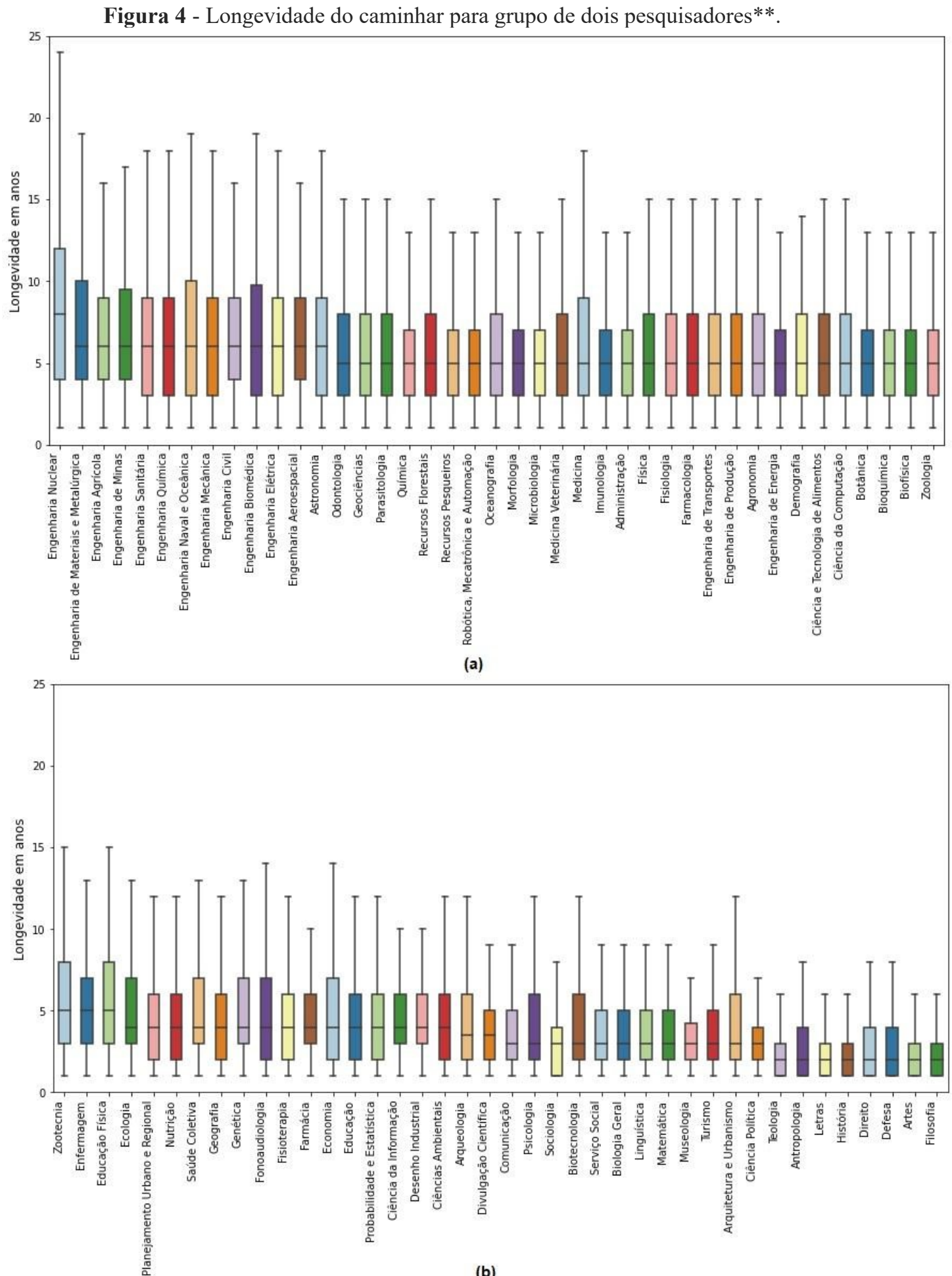

(b)

** A figura foi dividida em duas partes para permitir visualização dos resultados de todas as áreas analisadas (ordenadas a partir da maior mediana, desconsiderando os outliers). Fonte: Elaborada pelos autores.

Na Figura 4a é possível observar que a área Engenharia Nuclear com apenas 298 pesquisadores e 19.802 publicações (Tabela 1) possui a maior 
mediana de caminhares para dois pesquisadores ( 8 anos). Já a área de Medicina, que conta com 11.564 pesquisadores e 616.380 publicações, apresenta mediana no caminhar de dois pesquisadores de 5 anos. No entanto, cabe destacar, que a área também apresenta o maior caminhar (de 46 anos para grupo de dois pesquisadores) entre os 218.048 pesquisadores analisados que, por se tratar de um caminhar muito longo se comparado aos demais, é considerado um outlier, e não aparece representado na Figura 4a. A área de Medicina também se destaca em relação às outras áreas analisadas por conter caminhares acima de 15 anos para o grupo de dois pesquisadores (considerados outliers e não representados nas figuras).

A mediana de caminhares para o grupo de dois pesquisadores varia entre dois a oito anos. A única área com mediana de caminhares de oito anos é Engenharia Nuclear, seguida pelas áreas de Engenharia Aeroespacial, Engenharia Agrícola, Engenharia Biomédica, Engenharia Civil, Engenharia Elétrica, Minas e Astronomia (mediana de seis anos).

As áreas com as menores medianas são Antropologia, Artes, Defesa, Direito, Filosofia, História, Letras e Teologia (Figura 4b). À medida que aumenta o número de pesquisadores que publicam em colaboração, a mediana do caminhar diminui. Nestas áreas, para o grupo de dois pesquisadores a mediana é de oito anos, para três pesquisadores é de cinco anos. A partir do grupo de sete pesquisadores a mediana é de um ano, exceto para os grupos de 24 até 29 pessoas para os quais esse valor é de 1,5 anos.

$\mathrm{Na}$ Tabela 2 mostramos o maior caminhar por área para os menores e maiores grupos analisados. Os menores grupos são representados pelas colunas dois, três, quatro, cinco e seis, em que cada uma delas pode-se verificar para a área referida a quantidade em anos do maior caminhar encontrado nesses grupos de pesquisadores.

A área de Medicina, por exemplo, tem o maior caminhar para um grupo de dois pesquisadores de 46 anos e para um grupo de seis pesquisadores, caminhar de 21 anos. Aqui, não estamos representando quantas vezes ocorreu o caminhar com essa longevidade. Já entre os pesquisadores da área de Defesa, a 
maior longevidade do caminhar para grupo de dois pesquisadores é de onze anos e de três anos para grupo de seis pesquisadores (Tabela 2).

Tabela 2 - O maior caminhar dos pesquisadores por área.

\begin{tabular}{|c|c|c|c|c|c|c|c|c|}
\hline & & & & & & & & \\
\hline Área & Dois & Três & Quatro & Cinco & Seis & 38 & 39 & 40 \\
\hline Medicina & 46 & 34 & 26 & 24 & 21 & 2 & 1 & 1 \\
\hline Farmacologia & 42 & 22 & 17 & 8 & 5 & 1 & 1 & 1 \\
\hline Morfologia & 42 & 20 & 13 & 10 & 7 & 1 & 1 & 1 \\
\hline Geociências & 41 & 32 & 16 & 11 & 9 & 1 & 1 & 1 \\
\hline Enfermagem & 40 & 19 & 13 & 10 & 7 & 1 & 1 & 1 \\
\hline Odontologia & 40 & 31 & 22 & 20 & 9 & 1 & 1 & 0 \\
\hline Genética & 39 & 27 & 23 & 16 & 8 & 1 & 1 & 1 \\
\hline Bioquímica & 38 & 23 & 13 & 9 & 8 & 1 & 1 & 1 \\
\hline Física & 37 & 23 & 21 & 17 & 12 & 1 & 1 & 1 \\
\hline Zoologia & 36 & 16 & 10 & 8 & 7 & 1 & 1 & 1 \\
\hline Agronomia & 34 & 27 & 17 & 10 & 10 & 1 & 1 & 1 \\
\hline Fonoaudiologia & 34 & 24 & 15 & 12 & 9 & 1 & 1 & 0 \\
\hline Medicina Veterinária & 34 & 21 & 14 & 10 & 7 & 1 & 1 & 1 \\
\hline Microbiologia & 34 & 27 & 14 & 10 & 8 & 1 & 1 & 1 \\
\hline Parasitologia & 34 & 17 & 14 & 12 & 8 & 1 & 1 & 1 \\
\hline Recursos Pesqueiros e Engenharia de Pesca & 34 & 15 & 13 & 8 & 4 & 1 & 1 & 1 \\
\hline Engenharia de Materiais e Metalúrgica & 33 & 22 & 15 & 9 & 5 & 1 & 1 & 1 \\
\hline Engenharia Mecânica & 33 & 20 & 13 & 10 & 8 & 1 & 0 & 0 \\
\hline Engenharia Química & 33 & 18 & 11 & 8 & 5 & 0 & 0 & 0 \\
\hline Recursos Florestais & 33 & 22 & 9 & 7 & 6 & 1 & 1 & 1 \\
\hline Saúde Coletiva & 33 & 29 & 15 & 9 & 7 & 1 & 1 & 1 \\
\hline Direito & 32 & 28 & 15 & 10 & 7 & 1 & 1 & 1 \\
\hline Probabilidade e Estatística & 32 & 18 & 12 & 7 & 6 & 1 & 1 & 0 \\
\hline Engenharia Elétrica & 31 & 19 & 15 & 9 & 6 & 1 & 1 & 1 \\
\hline Fisiologia & 31 & 21 & 12 & 11 & 10 & 1 & 1 & 1 \\
\hline Astronomia & 30 & 30 & 14 & 11 & 8 & 2 & 1 & 1 \\
\hline Educação & 30 & 20 & 13 & 11 & 8 & 1 & 1 & 1 \\
\hline Engenharia Nuclear & 30 & 18 & 11 & 10 & 7 & 1 & 1 & 1 \\
\hline Farmácia & 30 & 18 & 16 & 10 & 6 & 1 & 1 & 1 \\
\hline Química & 30 & 27 & 21 & 10 & 7 & 1 & 1 & 1 \\
\hline Biofísica & 29 & 14 & 12 & 9 & 6 & 1 & 1 & 1 \\
\hline Botânica & 29 & 18 & 11 & 9 & 6 & 1 & 1 & 1 \\
\hline Ciência da Computação & 29 & 19 & 14 & 11 & 7 & 1 & 1 & 1 \\
\hline Ciência e Tecnologia de Alimentos & 29 & 24 & 16 & 12 & 6 & 1 & 1 & 0 \\
\hline Imunologia & 29 & 24 & 15 & 11 & 8 & 1 & 1 & 1 \\
\hline Psicologia & 29 & 18 & 15 & 9 & 9 & 1 & 1 & 1 \\
\hline Engenharia Biomédica & 28 & 18 & 13 & 10 & 6 & 0 & 0 & 0 \\
\hline Engenharia Civil & 28 & 20 & 12 & 9 & 8 & 1 & 0 & 0 \\
\hline Engenharia de Produção & 28 & 17 & 9 & 6 & 4 & 1 & 1 & 0 \\
\hline Robótica, Mecatrônica e Automação & 28 & 18 & 11 & 4 & 4 & 0 & 0 & 0 \\
\hline Zootecnia & 28 & 21 & 18 & 9 & 8 & 1 & 1 & 1 \\
\hline Arquitetura e Urbanismo & 27 & 13 & 9 & 7 & 6 & 0 & 0 & 0 \\
\hline Engenharia Agrícola & 27 & 20 & 13 & 11 & 10 & 0 & 0 & 0 \\
\hline Matemática & 27 & 14 & 11 & 7 & 7 & 1 & 1 & 1 \\
\hline Oceanografia & 27 & 18 & 9 & 7 & 5 & 1 & 1 & 1 \\
\hline Serviço Social & 27 & 10 & 8 & 5 & 5 & 1 & 1 & 1 \\
\hline Ecologia & 26 & 15 & 11 & 9 & 8 & 1 & 1 & 1 \\
\hline Biologia Geral & 25 & 19 & 12 & 9 & 8 & 1 & 1 & 1 \\
\hline Educação Física & 25 & 15 & 14 & 12 & 7 & 1 & 1 & 0 \\
\hline Engenharia Sanitária & 25 & 19 & 13 & 10 & 7 & 0 & 0 & 0 \\
\hline Nutrição & 25 & 16 & 12 & 8 & 6 & 1 & 1 & 1 \\
\hline Administração & 24 & 17 & 9 & 8 & 5 & 1 & 1 & 1 \\
\hline Desenho Industrial & 24 & 17 & 7 & 5 & 4 & 0 & 0 & 0 \\
\hline Economia & 24 & 14 & 9 & 7 & 5 & 1 & 1 & 1 \\
\hline Engenharia Aeroespacial & 24 & 12 & 10 & 7 & 6 & 1 & 1 & 1 \\
\hline História & 24 & 13 & 10 & 6 & 5 & 0 & 0 & 0 \\
\hline Comunicação & 23 & 11 & 6 & 5 & 5 & 1 & 1 & 1 \\
\hline Engenharia de Energia & 23 & 13 & 11 & 8 & 5 & 1 & 1 & 1 \\
\hline Antropologia & 22 & 11 & 7 & 4 & 4 & 1 & 1 & 1 \\
\hline Arqueologia & 22 & 13 & 9 & 5 & 4 & 0 & 0 & 0 \\
\hline Filosofia & 22 & 14 & 7 & 6 & 4 & 1 & 1 & 0 \\
\hline Letras & 22 & 17 & 9 & 8 & 8 & 1 & 1 & 0 \\
\hline Ciência Política & 21 & 10 & 7 & 4 & 3 & 1 & 1 & 1 \\
\hline Geografia & 21 & 13 & 9 & 7 & 5 & 1 & 1 & 1 \\
\hline Artes & 20 & 8 & 7 & 5 & 5 & 1 & 0 & 0 \\
\hline Biotecnologia & 20 & 13 & 10 & 8 & 6 & 1 & 1 & 1 \\
\hline Ciência da Informação & 20 & 13 & 9 & 9 & 6 & 0 & 0 & 0 \\
\hline Fisioterapia & 20 & 14 & 11 & 9 & 8 & 1 & 1 & 0 \\
\hline Planejamento Urbano e Regional & 20 & 14 & 7 & 5 & 4 & 1 & 1 & 0 \\
\hline Engenharia Naval e Oceânica & 19 & 10 & 7 & 5 & 3 & 0 & 0 & 0 \\
\hline Linguística & 19 & 14 & 7 & 6 & 5 & 1 & 1 & 1 \\
\hline Sociologia & 19 & 13 & 9 & 7 & 5 & 1 & 0 & 0 \\
\hline Teologia & 19 & 6 & 6 & 4 & 4 & 0 & 0 & 0 \\
\hline Ciências Ambientais & $\square 18$ & 18 & 10 & 6 & 4 & 1 & 1 & 1 \\
\hline Demografia & $\square 18$ & 9 & 6 & 4 & 4 & 0 & 0 & 0 \\
\hline Engenharia de Minas & $\square 18$ & 14 & 9 & 4 & 4 & 0 & 0 & 0 \\
\hline Engenharia de Transportes & 18 & 12 & 9 & 5 & 3 & 0 & 0 & 0 \\
\hline Turismo & $\square 18$ & 9 & 8 & 3 & 2 & 1 & 1 & 0 \\
\hline Divulgação Científica & $\square 14$ & 8 & 6 & 6 & 4 & 0 & 0 & 0 \\
\hline Museologia & $\square 14$ & 8 & 5 & 4 & 3 & 0 & 0 & 0 \\
\hline Defesa & $\square 11$ & 8 & 5 & 5 & 3 & 0 & 0 & 0 \\
\hline
\end{tabular}

Fonte: Elaborada pelos autores. 
Os maiores grupos analisados são representados pelas colunas 38, 39 e 40 (Tabela 2) e seguem a mesma lógica dos menores grupos, com a diferença que neste caso, a cor vermelha representa os maiores caminhares que seguem suavizando até chegar à cor branca.

Percebemos nessas três últimas colunas, que a quantidade em anos que os grupos maiores publicam juntos é bem menor e que o maior valor encontrado foi o caminhar de dois anos para grupos de 38 pessoas para as áreas de Medicina e Astronomia. De modo geral, para esses grupos de 38 a 40 pesquisadores, o maior caminhar encontrado foi de um ano. Outras 33 áreas não apresentaram grupo de 40 pesquisadores, por isso, não houve caminhar registrado.

A área Ciência da Informação (CI) conta com 914 pesquisadores doutores ativos cadastrados na Plataforma Lattes e 41.467 publicações, dentre as quais, 31.878 foram analisadas, sendo 9.586 publicações de autoria única (Tabela 1), e 3 publicações descartadas por apresentarem número maior que 40 coautores.

A Figura 5 mostra os caminhares para os pesquisadores da área de Ciência da Informação. O maior grupo que colaborou junto em uma publicação foi de 32 pesquisadores com caminhar de um ano e somente uma ocorrência. Podemos notar que a mediana da longevidade do caminhar é de quatro anos para grupo de dois pesquisadores e de dois anos para grupo de três pesquisadores.

Podemos ver na Figura 5 que para grupos de dois a dez pesquisadores os maiores caminhares são outliers. A partir do grupo de onze pesquisadores, o maior caminhar é de um ano, que é a mediana de cada um desses grupos e sem presença de outliers.

$\mathrm{Na}$ área da Ciência da Informação o caminhar mais longevo foi de 20 anos para grupo de dois pesquisadores e ocorreu apenas uma vez. Para o grupo de três pesquisadores o maior caminhar foi de 13 anos, para quatro e cinco pesquisadores o maior caminhar foi de 9 anos, e para o grupo de seis pesquisadores, o maior caminhar foi de seis anos. Para os grupos de sete, oito e nove pesquisadores o maior caminhar foi de três anos e de dois anos para o grupo de dez pesquisadores. 
Figura 5 - Longevidade do caminhar para os pesquisadores da área da Ciência da Informação.

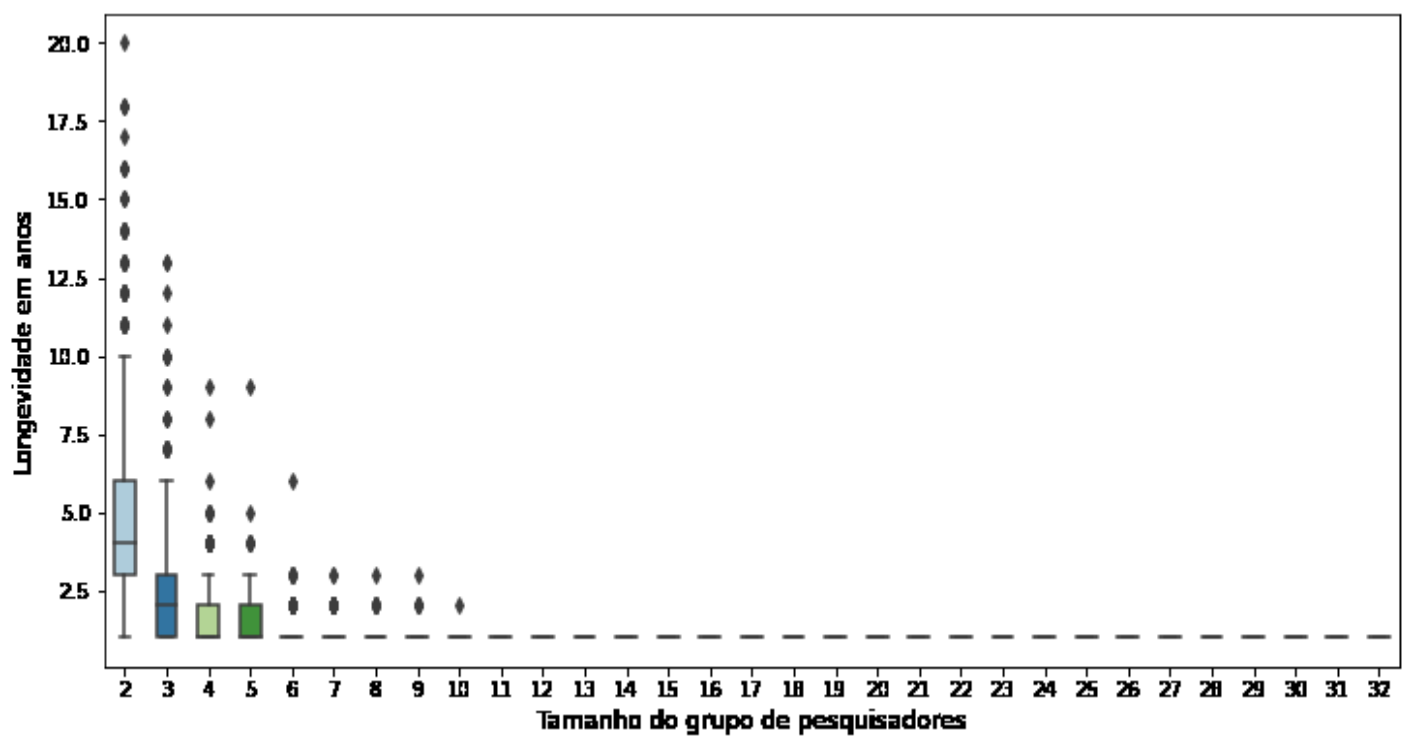

Fonte: Elaborada pelos autores.

Como vimos ocorrer em outras áreas, à medida que aumenta o tamanho do grupo de pesquisadores da área da CI, diminui a longevidade do caminhar.

Dentre os 914 pesquisadores da área da Ciência da Informação, o maior número de coautores com os quais um pesquisador colaborou ao longo de sua vida acadêmica foi de 243 coautores. Esse pesquisador teve 323 publicações analisadas, das quais 58 publicações de autoria única foram descartadas. Nas 314 publicações consideradas, o pesquisador trabalhou com no máximo 9 coautores em uma publicação.

Com o algoritmo proposto, para esse pesquisador foram geradas $\mathbf{1 . 5 6 8}$ combinações de coautores, pois conforme mostramos na Seção 2.4, com o nosso método garantimos que cada combinação formada contenha no mínimo uma publicação. Pelo método tradicional de agrupamento, considerando todos os possíveis grupos de 2 a 9 coautores entre os 244 pesquisadores (autor principal mais os 243 coautores), o total de agrupamentos seria 7.563.458.307.356.469 (i.e, mais de 7 quatrilhões), totalmente inviável de ser executado.

\section{Considerações finais}

Até o momento, a maior parte dos estudos realizados concentrou-se no estudo da colaboração a partir das publicações, isto é, na caracterização da área ou do 
pesquisador a partir das produções indexadas em uma fonte de dados, utilizando medidas quantitativas, como quantidade de artigos publicados. O tamanho do grupo de pesquisadores e o tempo de colaboração foram avaliados de forma independente. No presente estudo nos dedicamos a analisar a forma de interação dos pesquisadores através da longevidade das colaborações em anos, assim como o tamanho dos grupos de autores e coautores na colaboração científica por área do conhecimento.

Uma limitação deste trabalho se deve ao fato de termos optado por considerar apenas quatro tipos de publicações: artigos em periódicos, artigos em congressos, livros e capítulos de livros. A atuação acadêmica e a interação em pesquisas muitas vezes não são observadas e nem documentadas, como por exemplo, a participação em bancas, a orientação acadêmica, atividades de educação e popularização da ciência (entrevistas, participações em programas e comentários na mídia). O método proposto neste artigo pode ser empregado para qualquer conjunto de dados desde que haja registrada a interação, como no caso da coautoria.

Gerar todos os agrupamentos possíveis para pesquisadores de maior idade acadêmica, que durante sua vida acadêmica publicaram com mais de 100 diferentes coautores e em grupos de dez ou mais colaboradores foi um problema computacional que desafiou os autores e não pode ser resolvido em tempo hábil para esta pesquisa. Ainda assim, no presente trabalho foi possível propor um método para gerar os agrupamentos de coautores de forma eficiente e eficaz, onde somente foram computados (i.e., criados grupos) coautores que tinham no mínimo uma publicação. Essa caracterização foi analisada por idade acadêmica e por área do conhecimento, através de análise quantitativa. Os resultados apresentados mostram que existem diferenças de longevidade de caminhares entre as áreas analisadas, tanto em relação à idade acadêmica quanto à área de pesquisa. De forma geral, os grupos menores tendem a ter um caminhar mais longo em relação aos grupos maiores.

No trabalho de WANG et al. (2019), os autores investigaram se era possível prever/identificar colaboradores vitalícios, isto é, com um tempo de colaboração de 30 anos juntos. Conforme podemos observar na Tabela 2, 80\% 
das áreas estudadas têm caminhar com duração de 38 anos e podem ser consideradas áreas com colaborações vitalícias. Foi possível identificar também que dentre as 81 áreas analisadas, a área de Medicina apresentou o caminhar mais longevo (46 anos) para um grupo de dois pesquisadores.

De modo geral, as áreas da Saúde se destacaram com caminhares mais longos (Medicina, Farmacologia, Enfermagem, Odontologia), seguidas pelos pesquisadores das Engenharias e Ciências Exatas (Engenharia de Materiais e Metalúrgica, Engenharia Mecânica, Engenharia Química). Já as áreas Humanas e Sociais Aplicadas apresentaram caminhares mais curtos (História, Letras, Teologia, Defesa), embora a área de Direito se destacou com caminhar de longevidade de 32 anos. Foi possível observar também, que a área da Educação, (188.815 publicações) e a área de Letras (134.861 publicações) têm marcada característica de publicação de autoria única, o que explica estas áreas apresentarem caminhares curtos, uma vez que há pouca colaboração na forma de coautoria entre os pesquisadores.

Ao construir redes egocêntricas de pesquisadores com base na idade acadêmica, Lu et al. (2021) demonstraram que conforme a idade acadêmica dos pesquisadores aumenta, são formuladas trajetórias de crescimento positivas ou negativas dos papéis relativos ao longo de seus estágios de carreira, que são definidos como "padrões relativos de crescimento".

Nesse trabalho, também analisamos os caminhares dos pesquisadores por idade acadêmica (Figura 3) e foi possível observar para os tamanhos de grupos analisados que os pesquisadores com maior idade acadêmica tendem a ter um caminhar mais longo em relação aos pesquisadores com menor idade acadêmica, assim corroborando os achados de $\mathrm{Lu}$ et al. (2021). Futuramente, se pretende investigar melhor quais são os grupos que colaboram por mais tempo juntos e se há alguma influência da relação de orientador e orientado.

Ainda no que concerne à realização de trabalhos futuros, pretendemos realizar experimentos de maior profundidade analisando a longevidade de caminhares acadêmicos, suas especificidades e maior diversidade nas áreas de Ciência da Informação e Ciência da Computação. Trabalhos similares empregando a mesma metodologia podem ser realizados no contexto de outras 
bases de dados, como por exemplo, a base Digital Bibliograph \& Library Project (DBLP). Adicionalmente, podemos utilizar técnicas de mineração de dados, para categorizar os pesquisadores e poder definir padrões ao longo do tempo no nível de pessoas, grupos de pesquisa e instituições. Propomos ainda ponderar a longevidade pela intensidade da parceria, ou seja, analisar a longevidade em função do número de publicações no mesmo ano e, também, por quantidade de anos. Desse modo, além do tempo do caminhar em anos, podemos analisar também a quantidade de publicações realizadas durante o caminhar.

Outra possibilidade para continuidade desta pesquisa seria a utilização de períodos para contagem de tempo ao invés de ano. Além de verificar com quem um pesquisador colabora e a duração da colaboração, é importante também verificar se a colaboração é interdisciplinar, se as parcerias multidisciplinares são potencialmente mais longas.

A partir da geolocalização de cada coautor (latitude e longitude referente ao endereço profissional), é possível também analisar as distâncias geográficas para medir a descentralização geográfica da colaboração, enriquecendo ainda mais as análise e caracterização dos pesquisadores e das áreas.

Por fim, ressaltamos também como proposta futura a análise não somente da publicação individual, mas também institucional, nacional e internacional. E com isso mensurar se as parceiras mais longevas se dão nas colaborações nacionais ou internacionais, permitindo obter insumos para analisar em maior profundidade a internacionalização e interdisciplinaridades dos caminhares acadêmicos.

\section{Referências}

ADAMS, Jonathan. Collaborations: the rise of research networks. Nature, [s.1.], v. 490, n. 7420, p. 335-336, 2012.

BOUKERCHE, Azzedine; ZHENG, Lining; ALFANDI, Omar. Outlier Detection: Methods, Models, and Classification. ACM Computing Surveys, [s.1], v. 53, n. 3, 2020. 
DIGIAMPIETRI, Luciano Antonio; TUESTA, Esteban Fernandez; KÖHLER, André Fontan; DELGADO, Karina Valdivia; BERNARDES JÚNIOR, João Luiz. Caracterizando o processo de doutoramento no Brasil ao longo dos anos: período de formação, sexo e produção acadêmica. Em Questão, Porto Alegre, v. 27, n. 1, p. 361-387, jan/abr. 2021.

GAO, Ying; XIAOQIANG, Wu; WEI, Yan; ZHANG, Lei; TUNHUA, Wu. Dynamic network embedding enhanced advisor-advisee relationship identification based on internet of scholars. Future Generation Computer Systems, [s.1.] v. 108, p. 677-686, 2020.

HAINES, Valerie A.; GODLEY, J.; HAWE, Penelope. Understanding Interdisciplinary Collaborations as Social Networks. American Journal of Community Psychology, [s.1], v. 47, p. 1-11, 2011.

KATZ, J. Sylvan.; MARTIN, Ben R. What is research collaboration? Research Policy, [s.1.], v. 26, n. 1, p. 1-18, 1997.

LANE, Julia. Let's make science metrics more scientific. Nature, [s.1.], v. 464, p. 488-489, 2010.

LETA, Jacqueline; THIJS, Bart; GLÄNZEL, Wolfgang. A macro-level study of science in Brazil: seven years later. Encontros Bibli: Revista Eletrônica de Biblioteconomia e Ciência da Informação, Florianópolis, v. 18, n. 36, p. 5166, 2013.

LEYDESDORFF, Loet; WAGNER, Caroline; PARK, Han Woo; ADAMS, Jonathan. International collaboration in science: the global map and the network. El profesional de la información, [.s], v. 22, n. 1, p. 87-95, 2013.

LU, Wei; REN, Yan; HUANG, Yong; BU, Yi; ZHANG, Yuehan. Scientific collaboration and career stages: An ego-centric perspective. Journal of Informetrics, [s.1], v. 15, p. 101-217, n. 4, 2021.

PLATAFORMA LATTES. Extração de Dados. 2020. Disponível em: http://memoria.cnpq.br/web/portal-lattes/extracoes-de-dados.Acesso em: 1 mar. 2020.

SIDONE, Otávio José Guerci; HADDAD, Eduardo Amaral; MENA-CHALCO, Jésus Pascual. A ciência nas regiões brasileiras: evolução da produção e das redes de colaboração científica. Transinformação, Campinas, v. 28, n. 1, p. 15$32,2016$.

THE ROYAL SOCIETY. Knowledge, networks and nations: global scientific collaboration in the 21 st century. Londres: The Roya Society, 2011.

TSAI, Chun-Hua; LIN, Yu-Ru. Tracing and Predicting Collaboration for Junior Scholars. In: Proceedings of the 25th International Conference Companion on 
World Wide Web, 16, 2016, Canada. WWW'16, Canada: International World Wide Web Conferences Steering Comettee, n. 6, p. 375-380, 2016.

TUESTA, Esteban Fernandez.; DELGADO, Karina. Valdivia; MUGNAINI, Rogério; DIGIAMPIETRI, Luciano Antonio; MENA-CHALCO, Jesús Pascual; PÉREZ-ALCÁZAR, José J. Analysis of advisor-advisee relationship: an exploratory study of the area of exact and Earth Sciences in Brazil. PLOS One, San Francisco, v. 10, n. 5, e 0129065, 2015.

TUESTA, Esteban Fernandez; DIGIAMPIETRI, Luciano Antonio; DELGADO, Karina Valdivia; MARTINS, Nathália Ferraz Alonso. Análise da participação das mulheres na ciência: um estudo de caso da área de ciências exatas e da terra no brasil. Em Questão, Porto Alegre, v. 25, n. 1, p. 37-62, 2019.

VANZ, Samile Andrea de Souza; STUMPF, Ida Regina Chittó. Colaboração científica: revisão teórico-conceitual. Perspectivas em Ciência da Informação, Minas Gerais, v. 15, n. 2, p. 42-55, 2010.

WANG, Wei; WAN, Liangtian; KONG, Xiangjie; GONG, Zhiguo; XIA, Feng. Not Every Couple Is a Pair: A Supervised Approach for Lifetime Collaborator Identification. In: Pacific Asia Conference on Information Systems, 23, 2019, China. PACIS 2019, China: Association for Information, 2019.

\title{
Analysis of longevity and size of academic co-authorships: the walk in the Brazilian science
}

\begin{abstract}
This article offers a method of detection, identification and visualization of longevity (time) and co-authorship size (number of people) in Brazilian Science, which makes it possible to analyze the paths of researchers according to knowledge areas. The data used for all active PhDs in 2019 registered in the Lattes Platform. The method allowed us to observe over time all possible research groups in the co-authorship collaboration. The results provide evidence that smaller groups have a longer lifespan compared to larger size groups. This article highlights in unprecedented way the paths (due to longevity and size) of Brazilian researchers, allowing to observe the performance of each researcher not in an isolated / independent way, but in a group way / dependent on collaborative and social interaction, as Science is performed nowadays.
\end{abstract}

Keywords: Scientific collaboration; Co-authorship; Longevity. Academic walk. 


\section{Análisis de la longevidad y del tamaño de las coautorías académicas: los caminares en la ciencia brasileña}

Resumen: Este artículo propone un método de detección, identificación y visualización de la longevidad (tiempo) y el tamaño de la coautoría (número de personas) en la ciencia brasileña, que permite analizar los caminos de los investigadores según áreas de conocimiento. Los datos utilizados se refieren a todos los doctores activos en 2019 registrados en la Plataforma Lattes. El método utilizado nos permite observar todos los posibles grupos de investigadores en la colaboración, en términos de coautoría, a lo largo del tiempo. Los resultados presentados muestran que los grupos más pequeños tienen una vida útil más larga en comparación con los grupos de mayor tamaño. Este artículo caracteriza de manera inédita los caminares (por longevidad y tamaño) de los investigadores brasileños, permitiendo observar el desempeño de cada investigador, no de manera aislada/independiente y si de forma grupal/dependiente de la interacción colaborativa y social, como es realizada la ciencia en la actualidad.

Palabras clave: Colaboración científica; Coautoría; Longevidad; Caminar académico

Recebido: 22/06/2021

Aceito: $17 / 09 / 2021$

\section{Declaração de autoria}

Concepção e elaboração do estudo: A.C.S Gusmão, J.P. Mena-Chalco

Coleta de dados: A.C.S. Gusmão.

Análise e interpretação de dados: A.C.S. Gusmão, S.M. Santos, J.P. Mena-

Chalco.

Redação: A.C.S. Gusmão, S.M. Santos, J.P. Mena-Chalco.

Revisão crítica do manuscrito: A.C.S. Gusmão, S.M. Santos, J.P. Mena-

Chalco.

\section{Como citar:}

GUSMÃO, Andréia Cristina dos Santos; SANTOS, Solange Maria dos; MENACHALCO, Jesús Pascual. Análise da longevidade e do tamanho das coautorias acadêmicas: os caminhares na ciência brasileira. Em Questão, Porto Alegre, v. 28 , n. 2, e-116156, abr./jun. 2022. https://doi.org/10.19132/18085245282.116156

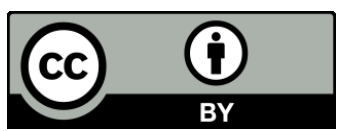

